and valproic acid may have contributed to the movement disorder. In case 2, receiving phenytoin, a gabapentin rechallenge caused recurrence of choreoathetosis in 7 days but to a lesser degree; the reduced severity of movements was related to a reduction in dosage of phenytoin. (Chudnow RS, Dewey RB Jr, Lawson CR. Choreoathetosis as a side effect of gabapentin therapy in severely neurologically impaired patients. Arch Neurol July 1997;54:910912). (Respond: Robert S Chudnow MD, Department of Neurology, University of Texas Southwestern Medical Center, 1935 Motor St, Dallas, TX 75235).

COMMENT. The risk of gabapentin-associated choreoathetosis in this institution was 2 of 28 patients treated $(7.1 \%)$. The risk appears to be related to polytherapy and high serum levels of other anticonvulsants, VPA and phenytoin, also known to cause choreoathetosis. Neurological impairments and intractable epilepsy with brain damage are likely contributing factors. A previous report of choreoathetosis with gabapentin in a severely mentally retarded adult is reviewed in Progress in Pediatric Neurology III, 1997, p157.

\title{
ANEMIA ASSOCIATED WITH LAMOTRIGINE
}

Two cases of anemia associated with lamotrigine adjunctive therapy for intractable epilepsy, one a 17-year-old with Lennox-Gastaut syndrome, are reported from Texas Tech University, Lubbock, TX. One patient had a previous history of iron deficiency anemia. The lowest Hct and $\mathrm{Hgb}$ levels, recorded after 2 months of treatment with lamotrigine, in combination with valproic acid, were Hct 32.7 and $18.9 \%$ and $\mathrm{Hgb} 9.9$ and $7 \mathrm{~g} / \mathrm{dl}$. An associated increase in platelets to 427 and $446 \mathrm{~K} / \mathrm{mcl}$ represented a reactive thrombocytosis. Neither case required intensive medical management, and the anemia resolved rapidly when the lamotrigine was discontinued. (Esfahani FE, Dasheiff RM. Anemia associated with lamotrigine. Neurology July 1997;49:306-307). (Reprints: Dr Richard M Dasheiff, Texas Tech University Health Sciences Center, Division of Neurology, 36014 th St, Lubbock, TX 79430).

COMMENT. The authors cite 3 previous case reports of hematological side effects with lamotrigine, all associated with polytherapy, including valproic acid or carbamazepine, drugs known to cause anemia or leukopenia. CBC monitoring may be advisable when introducing lamotrigine, particularly in patients receiving valproic acid or carbamazepine in combination.

\section{SEIZURE DISORDERS}

\section{POST-SURGICAL OUTCOME OF INFANTILE SPASMS}

Two-year postsurgical developmental outcome was assessed in 24 children with infantile spasms treated at the University of California, Los Angeles. All were symptomatic cases with neurological deficits and had received ACTH and multiple medications without benefit before the cortical resections. Seizures began at a mean age of 12 weeks and surgery was at 20 months of age. The developmental levels assessed by the Vineland Adaptive Behavior Scales were significantly increased at 2 years post surgery compared to presurgical levels, and only one child was severely retarded. The outcomes in this UCLA surgical series were equal to and sometimes superior to other symptomatic series receiving only medical treatment. Those who received surgery at an early age had the better presurgical developmental levels and the best 2-year postsurgery outcomes. (Asarnow RF, LoPresti C, Guthrie D et al. 